\title{
Enteroendocrine cell lineage revealed by time-resolved differentiation map
}

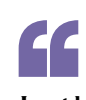

In the latest

study, the investigators

aimed to

provide a

definitive

lineage tree

of entero-

endocrine differ-

entiation...

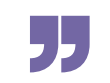

A new study published in Cell has mapped out the lineage tree of enteroendocrine cell differentiation using single-cell sequencing and a novel time-resolved reporter system. This work revealed hormonal plasticity during the maturation of these cells and identified drivers of individual enteroendocrine phenotypes that could be used for therapeutic manipulation.

The rapidly renewing intestinal epithelium features multiple cell types that all originate at the base of the intestinal crypt from stem cells. One of the progeny of these cells are the enteroendocrine cells, which are a hormone-producing population with roles in metabolism, appetite control, intestinal motility and immunity. Enteroendocrine cells are usually classified on the basis of the hormones they produce; however, reports of multihormonal cells exist, which has complicated the classification of what could be up to 20 different cell types.

"These findings posed the question of whether these multihormonal cells are evidence of independent lineages, of a general promiscuity in hormone expression or of enteroendocrine cell plasticity," explains lead author Helmuth Gehart. Although some regulators

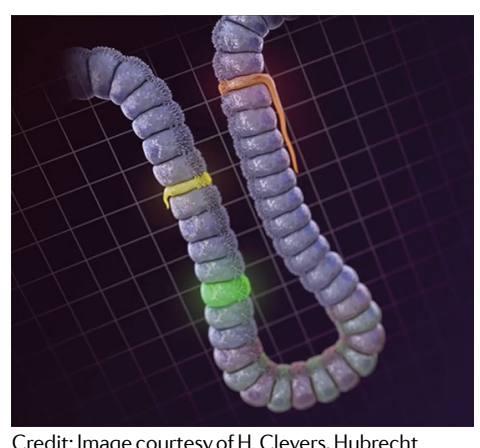

Credit: Image courtesy of H. Clevers, Hubrecht Institute, Utrecht, Netherlands of enteroendocrine development have been characterised, such as neurogenin-3 (NEUROG3), a complete map of the individual enteroendocrine lineages was previously missing. In the latest study, the investigators aimed to provide a definitive lineage tree of enteroendocrine differentiation to answer some of these questions.

The researchers used single-cell sequencing to examine the transcriptomes of thousands of enteroendocrine cells, but faced a challenge in finding a way to integrate the transcriptome data into a fate map. "We opted for a method that introduced an additional unbiased biological factor to guide our map assembly: real-time," says Gehart.

As enteroendocrine cells all express NEUROG3 as a pulse during their common progenitor phase, the team developed the Neurog3 Chrono mouse, which expresses a bi-fluorescent reporter under the control of Neurog3. When Neurog3 is pulsed on and off, green fluorescence rises quickly before vanishing, but it is then followed by red fluorescence that is detectable for days. "Thus, we can determine the exact time after the pulse of Neurog3 expression in each cell by measuring individual fluorescence," explains Gehart. By combining this system with single-cell transcriptomics, a lineage tree was built along a real-time axis with single-cell resolution.

"We could show that there are essentially six enteroendocrine lineages," reports Gehart. "However, to our surprise, many enteroendocrine cells switched the hormone they produced in the course of their lives." This finding showed that the hormonal capabilities of an individual enteroendocrine cell had limited plasticity and provided an explanation for the observed multihormonal cells. The investigators were also able to follow the movement of enteroendocrine cells across the crypt-villus axis and found that they remained in the crypts much longer than enterocytes. "Interestingly, the time at which many enteroendocrine cells left the crypt coincided with the observed cell type switches. This indicates that the hormonal plasticity is, at least in part, controlled by the positon of individual cells," explains Gehart.

Finally, the researchers used their transcriptomic data from cell types at different stages of differentiation to examine decision points along their lineage tree and identify candidate drivers for each fate. "We chose nine candidates and knocked them out in mice or intestinal organoids to prove the validity of our predictions," says Gehart. "Six of these nine gave strong enteroendocrine phenotypes, which demonstrates the power of our method to accurately unravel complex differentiation systems."

These data will enable a better understanding of enteroendocrine cells in homeostasis but also in the context of disease, as there are many disorders that could benefit from targeted therapeutic manipulation of these cells. "The next step will be to translate this knowledge and generate the tools that allow us to precisely shape the enteroendocrine landscape for basic research and therapy," concludes Gehart.

Iain Dickson

ORIGINAL ARTICLE Gehart, H. et al. Identification of enteroendocrine regulators by real-time singlecell differentiation mapping. Cell https://doi.org/ 10.1016/j.cell.2018.12.029 (2019) 